\title{
An unusual mixed germ cell tumor of the testis consisting of rhabdomyosarcoma, mature teratoma and yolk sac tumor
}

\author{
Eva Lovrić ${ }^{1}$, Dubravka Bobonj Hižak ${ }^{2}$, Melita Perić Balja ${ }^{3}$, Tanja Leniček ${ }^{4}$, Božo Krušlin ${ }^{4}$ \\ ${ }^{1}$ Department of Pathology and Cytology, Čakovec County Hospital, Čakovec 40000, Croatia \\ ${ }^{2}$ Department of Pathology, Bjelovar General Hospital, Bjelovar 43000, Croatia \\ ${ }^{3}$ Department of Pathology, University Hospital for Tumors, Zagreb 10000, Croatia \\ ${ }^{4}$ Ljudevit Jurak University Department of Pathology, Sestre milosrdnice University Hospital, Zagreb 10000, Croatia
}

Asian Journal of Andrology (2010) 12: 451-452. doi: 10.1038/aja.2010.2; published online 22 February 2010.

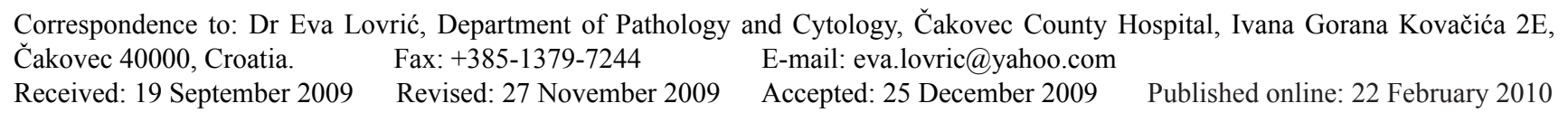

Dear Editor,

We recently encountered a rare case of testicular mixed germ cell tumor (MGCT) in a 32-year-old man. The tumor was composed of a combination of a yolk sac tumor, teratoma and rhabdomyosarcomatous somatic type malignancy.

The patient presented with left testicular swelling and pain. Physical examination, ultrasonography and computed tomography revealed a tumor in the left testicle. Alpha feto-protein (AFP) was elevated (720 $\mathrm{mIU} \mathrm{mL}^{-1}$; normal reference range: $0-9 \mathrm{mIU} \mathrm{mL}^{-1}$ ). A simple orchiectomy was performed.

Grossly, the tumor was tan and well circumscribed, measuring $7.0 \times 6.0 \times 4.5 \mathrm{~cm}^{3}$, showing a soft and yellow cut surface, with areas of cartilaginous tissue and only a slim margin of residual testicular tissue. Away from the main mass there was an additional wellcircumscribed nodule (12 $\mathrm{mm}$ in diameter) (Figure 1A).

Routine formalin-fixed, paraffin-embedded tissue sections were stained with hematoxylin and eosin. An additional immunohistochemical stain for desmin, myoglobin and myogenin (DAKO, Glostrup, Denmark) was performed on a representative section of the tumor.

Microscopically, the tumor was composed of a yolk sac tumor $(50 \%)$ and a mature teratoma (30\%) germ cell component associated with a somatic type embryonal rhabdomyosarcoma component $(20 \%)$. The size of the largest focus of the rhabdomyosarcoma component is $12 \mathrm{~mm}$. The yolk sac tumor showed a microcystic pattern, glandular structures, mesenchymal stroma, and partly micropapillary structures with perivascular Schiller-Duval bodies (Figure 1B). The teratoma was composed of well-differentiated cartilage and cystic formations surrounded with dense connective tissue stroma (Figure 1C). The rhabdomyosarcomatous component from a separate nodule showed sheets of atypical small cells with pleomorphic hyperchromatic nuclei and elongated cells with abundant eosinophilic cytoplasm (Figure 1D and E). The mitotic count was high (7/10 HPF). Immunohistochemically these cells strongly expressed desmin (Figure 1F), myogenin (Figure 1G) and myoglobin (Figure 1H). The adjacent testicular parenchyma contained foci of intratubular germ cell neoplasia of an unclassified type. Necrosis was observed in less than $10 \%$ of the tumor. No signs of vascular invasion and no spread to the rete testis, epididymis or beyond the tunica albuginea were found. Follow-up at 6 months after surgery did not show any recurrence or metastasis. The patient did not receive any additional therapy.

In the study by Bray et al. [1], the incidence of testicular cancer has shown a consistent increase in the European countries, while at the same time there was a decline in testicular cancer mortality. Advances in the treatment of testicular cancer caused large decreases in 

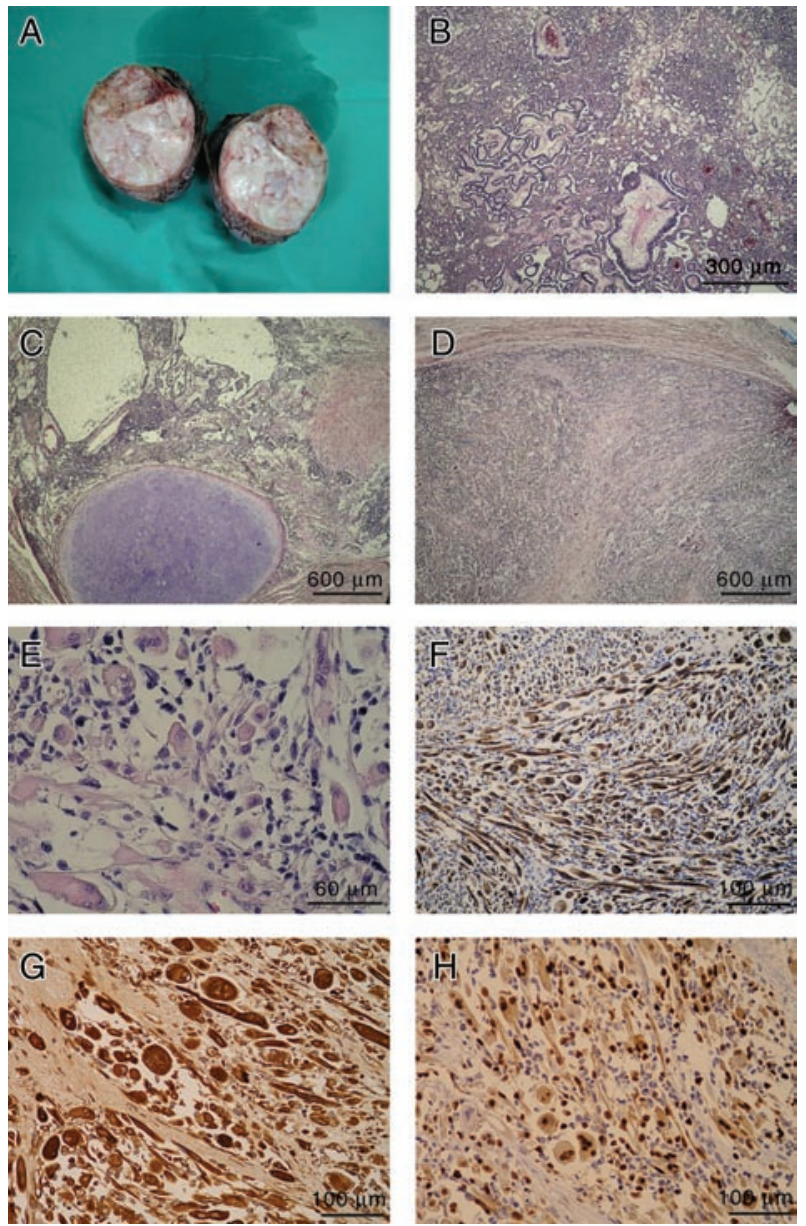

Figure 1. Grossly, the tumor was well circumscribed, showing a soft and yellow cut surface, with areas of cartilaginous tissue and with only a slim margin of residual testicular tissue. Away from the main mass there was an additional well-circumscribed nodule (A). Microscopically, the tumor was composed of a yolk sac component $(\mathrm{B}, 100 \times \mathrm{HE})$, mature teratoma $(\mathrm{C}, 40$ $\times \mathrm{HE})$, and rhabdomyosarcoma $(\mathrm{D}, 40 \times \mathrm{HE}$ and $\mathrm{E}, 400 \times)$. Immunohistochemically, the rhabdomyoblasts showed a positive reaction for desmin $(\mathrm{F}, \times 200)$, myogenin $(\mathrm{G}, \times 200)$ and myoglobin $(\mathrm{H}, \times 200)$.

mortality in some European countries, despite increases in incidence [1].

Testicular MGCTs represent $32 \%-54 \%$ of all germ cell tumors $[2,3]$. They usually occur at the mean age of 30 years. Clinical presentation includes painless or painful enlargement of the testis. Serum levels of AFP and beta-hCG are often elevated [2].

MGCTs consist of more than one histological com- ponent that may occur in any combination. Testicular germ cell tumors are omnipotent, can proliferate into homogeneous seminomas and are able to differentiate into any type of tissue, both in the embryo and outside, and form seminomas or nonseminomas (teratomas). The precursor lesion or carcinoma in situ cells show phenotypic similarity to primordial germ cells or gonocytes, which suggests that the cells were stopped at an early stage of differentiation [4].

The most common combination is teratoma and embryonal carcinoma [2]. A case of rhabdomyosarcoma in a mature teratoma and a case of rhabdomyosarcoma in a mediastinal teratoma have been reported [3, 5]. Nongerm cell malignant tumors of somatic type malignancy may arise in primary or metastatic germ cell tumors and are most likely derived from teratomas. They are seen in 3\%-6\% of patients with metastatic MGCTs, suggesting a poor prognosis [2].

MGCT of the testis in combination with the somatic type rhabdomyosarcoma that occurred in our patient is quite uncommon. Recognition of this variant is important because it may mimic a somatic sarcoma if inadequately sampled, and the clinical outcome may differ from the typical testicular MGCT. Therefore, correct histological analysis of testicular MGCTs and their metastases are essential parts of the diagnosis that will guide the correct therapeutic approach and give useful information on the prognosis.

\section{References}

1 Bray F, Richiardi L, Ekbom A, Pukkala E, Cuninkova M, et al. Trends in testicular cancer incidence and mortality in 22 European countries: continuing increases in incidence and declines in mortality. Int J Cancer 2006; 118: 3099-111.

2 Eble JN, Sauter G, Epstein JI, Sesterhenn IA (Eds.). World Health Organization Classification of Tumours. Pathology and Genetics of Tumours of the Urinary System and Male Genital Organs. Lyon: IARC Press; 2004. 217-46.

3 Korski K, Breborowicz D, Filas V, Breborowicz J, Grygalewicz B, et al. A case of primary testicular germ cell tumor with rhabdomyosarcoma metastases as an example of applying the FISH method to diagnostic pathology. APMIS 2007; 115: 1296-301.

4 Looijenga LH. Human testicular (non)seminomatous germ cell tumours: the clinical implications of recent pathobiological insights. J Pathol 2009; 218: 146-62.

5 Omezzine N, Khouatra C, Larive S, Freyer G, Isaac-Pinet S, et al. Rhabdomyosarcoma arising in mediastinal teratoma in an adult man: a case report. Ann Oncol 2002; 13: 323-6. 